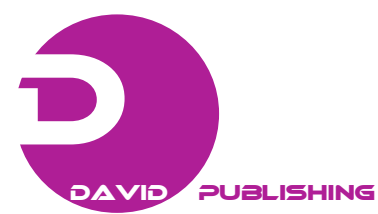

\title{
The Interaction of Prandtl-Meyer Wave with the Oblique Shock of the Same Direction
}

\author{
Vladimir Uskov ${ }^{1}$ and Mikhail Chernyshov ${ }^{2}$ \\ 1. Department of Plasma and Gas Dynamics, Baltic State Technical University, Saint Petersburg 190005, Russia \\ 2. Department of Extreme Phenomena in Materials and Blast Safety, Saint Petersburg State Polytechnical University, Saint
}

Petersburg 195251, Russia

Received: March 04, 2013 / Accepted: July 02, 2013 / Published: January 31, 2014.

\begin{abstract}
The interaction of the oblique stationary shock with the preceding Prandtl-Meyer expansion or compression wave is studied theoretically and numerically. Two analytical models are proposed for the flow description, which, though being approximate, gives us the solution of the problem with very high accuracy level. Owing to proposed analytical models, distinctive features of the flow in the interaction region, curvilinear shock inflection, the reflected expansion/compression wave type change, the degeneration of the resulted waves (for example, of the oblique shock into weak discontinuity), occurrence of the subsonic pockets downstream the interacting shock, are discovered and characterized analytically.
\end{abstract}

Key words: Supersonic flow, shock-expansion interaction, analytical models and solutions.

\section{Nomenclature}

$J_{i} \quad$ The strength of the $i$-th stationary shock of Prandtl-Meyer wave (i.e., the relation of the static pressures downstream and upstream it)

$j_{i} \quad i$-th stationary shock

$K_{\sigma} \quad$ Geometrical curvature of the shock

$M \quad$ Mach number

$N_{i} \quad i$-th "basic non-uniformity of the flow"

$n \quad$ Natural coordinate (the direction normal to the streamline)

$p \quad$ Static pressure

$p_{0} \quad$ Full (stagnation) pressure

$r_{i} \quad i$-th Prandtl-Meyer compression or expansion wave

$s \quad$ Natural coordinate (streamline direction)

$x, y \quad$ Horizontal and vertical directions

$\alpha \quad$ Mach angle

$\beta_{i} \quad$ Flow deflection angle on the $i$-th shock or Prandtl-Meyer wave

$\gamma \quad$ The ratio of gas specific heats

$\delta \quad$ Flow symmetry factor

$\varepsilon$ The function of specific heats ratio,

Corresponding author: Mikhail Chernyshov, Dr. Sc. (Engr.), professor, research fields: gas dynamics, shock interaction blast inhibitors. E-mail: chernyshov@npo-sm.ru.

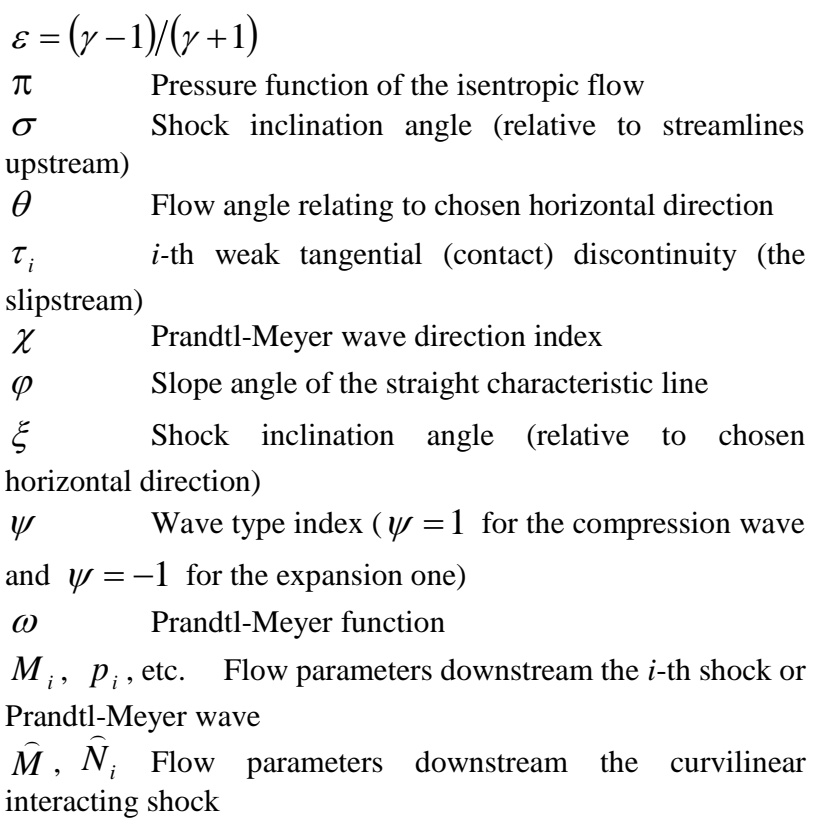

\section{Introduction}

The problems of interactions between Prandtl-Meyer waves and oblique stationary shocks can be treated as classical in the theory of discontinuities interactions in gas dynamics. Owing to the numerical methods, these problems can be solved 
in each separate case. But, in spite of the long history of studies [1], full theoretical analysis of the solution has not been executed yet. A lack of exact analytical relations as well as the complexity of the flow (the interaction is not localized at a single point and even in any finite region) is the main obstacles in the way of theoretical study.

With the problem to be solved now, an initially uniform gas stream with Mach number $M_{0}$ (Fig. 1) turns along the plane wall, and simple (non-centered, Figs. 1a and 1c) or centered (with the only common point of all straight acoustic characteristics, Figs. 1b and 1d) Prandtl-Meyer wave $r_{1}$ originates.

The oblique stationary shock $j_{2}$ of the same direction is situated downstream the isentropic wave. The supersonic gas flow in the region 1 between the wave $r_{1}$ and the shock $j_{2}$ can be characterized by its Mach number, $M_{1}$ and flow angle, $\theta_{1}$. Here, we admit that the flow angle $\theta_{1}=0$ (i.e., the flow direction is horizontal in the chosen coordinate system). The distance between the point $O$ of the intersection of the last Prandtl-Meyer straight characteristic $O B$ with the streamlined surface (in particular, $O$ is the center of the rarefaction wave in Fig. 1b), and the corner point $A$ of

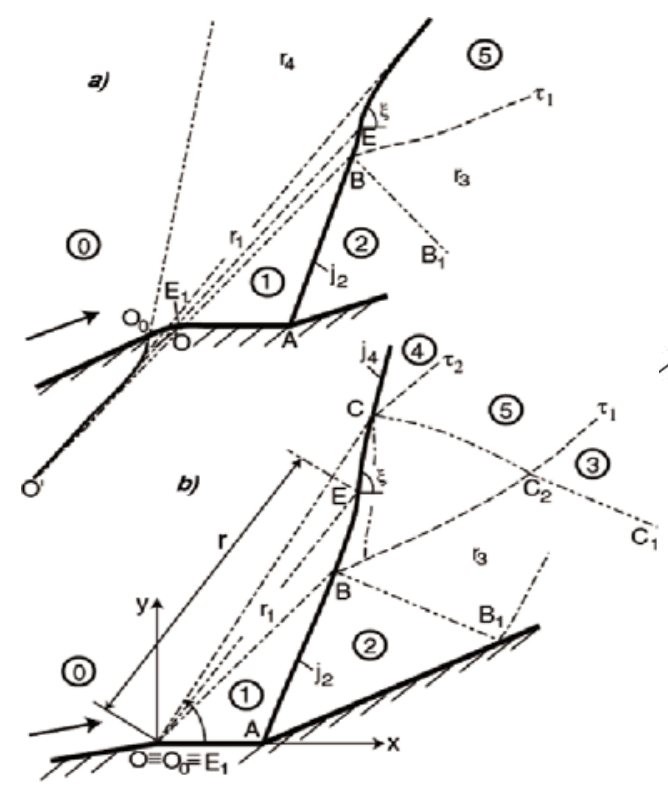

shock generation, is the length scale $|O A|=1$.

Initially, the straight shock $j_{2}$ becomes curvilinear at its intersection with the last characteristic $O B$ of the wave $r_{1}$. The reflected weak discontinuity $B B_{1}$ comes out at the point $B$, if the flow behind the shock $j_{2}$ is supersonic. This discontinuity is a border between the uniform flow region 2 behind the shock, and the reflected perturbations in the region $r_{3}$.

Due to contiguity theorem, the region $r_{3}$ is either a rarefaction or compression Prandtl-Meyer wave.

Weak tangential (contact) discontinuity $\tau_{1}$ ("weak" means the discontinuity of not flow parameters, but of their first spatial derivatives) originates from point $B$ downstream the shock. The discontinuity $\tau_{1}$ is the lower border of the substantially non-isentropic flow region 5 which can also be named the vortex layer, or the slipstream of finite width. Another weak tangential discontinuity $\tau_{2}$ which originates from the point $C$ of intersection between the shock and the first straight characteristic of the wave $r_{1}$ is the upper border of the vortex region 5 . The weak discontinuity $C C_{1}$ also goes out from the point $C$ and encloses the reflected wave $r_{3}$ if the flow downstream the shock is really supersonic at the point $C$.

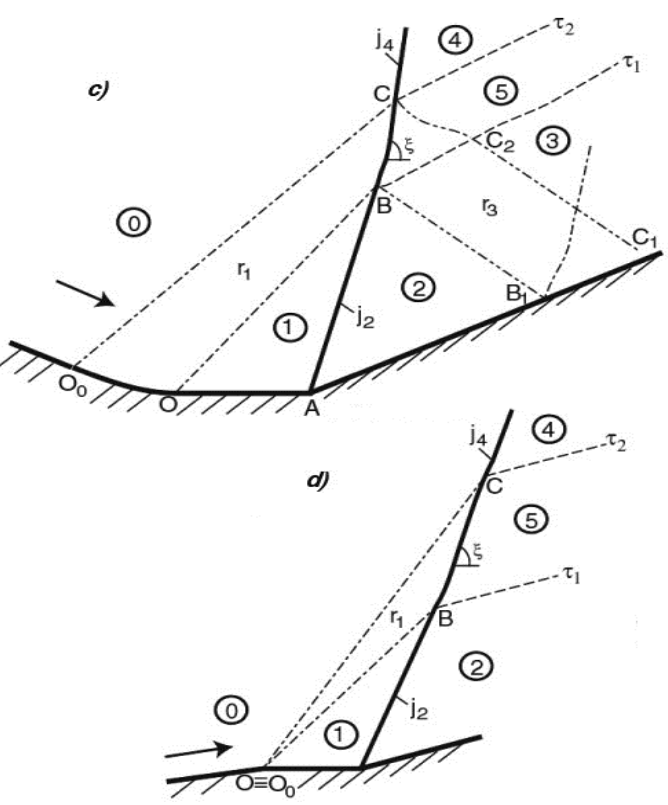

Fig. 1 Interaction of the Prandtl-Meyer wave with the proceeding oblique shock: (a) the asymptotic degeneration of the shock inside the expansion fan; (b) the shock interaction with the centered wave; (c) the shock strengthening under the compression wave $r_{1}$ influence; (d) the case of the subsonic flow downstream the shock $j_{2}$. 
If the Prandtl-Meyer expansion wave is too strong, then the curvilinear shock degenerates into one more weak discontinuity inside the expansion fan (Fig. 1a). The discontinuity $\tau_{2}$ does not exist there, and non-interacted part $r_{4}$ of the wave $r_{1}$ serves instead of the shock $j_{4}$ which comes through the expansion fan otherwise.

The oblique shock $j_{4}$ (Figs. 1b-1d) or the expansion wave $r_{4}$ (Fig. 1a) of the same direction as the wave $r_{1}$ and the shock $j_{2}$ (direction index $\chi=1$ ) as well as the reflected isentropic wave $r_{3}$ of the opposite direction $(\chi=-1)$ result due to the interaction considered. If the flow downstream the shock is subsonic, then the Prandtl-Meyer wave $r_{3}$ does not exist, and the results of interaction sufficiently depend upon the perturbation induced downstream. We do not consider the latter type of interaction, except of several complementary remarks.

We should emphasize that the wave $r_{3}$ is also partly a result of the refraction of the perturbations reflected from the shock in the region $B C C_{2}$ on the vortex flow layer. At this refraction, the perturbations reflected from the non-isentropic layer 5 are generated in their turn. These reflected perturbations distribute along the characteristics of the first family, overtake the shock situated upstream them, influence its features and shape, and make it curvilinear even after point $C$. That is why the shock $j_{4}$ can not be formally called straight, and the flow behind it (in the region 4) is not exactly uniform and isentropic.

The main goals of the present study are to define and analyze the shape and the other features of the interacting shock, to find out the type of the resulted perturbations and their transition criteria, as well as some special features of the considered interaction, and the influence of the ratio of gas specific heats on the problem solution.

\section{Materials and Methods}

2.1 Non-uniformities of the Two-Dimensional Gas Flow

To characterize not only flow parameters, but also their spatial derivatives at the non-uniform stream of a perfect inviscid gas, we introduce so-called in Ref. [2] "basic non-uniformities" given from two-dimensional flow equations in natural coordinates:

$$
\begin{gathered}
\frac{M^{2}-1}{\gamma M^{2}}+\frac{\partial \theta}{\partial n}+N_{4} \sin \theta=0, \\
\gamma M^{2} N_{2}=-\partial \ln p / \partial n, \\
\partial p_{0} / \partial s=0 .
\end{gathered}
$$

Here, $N_{1}=\partial \ln p / \partial s$ expresses the flow non-isobaricity, $N_{2}=\partial \theta / \partial s$ is the geometric curvature of streamlines, the quantity $N_{3}=\partial \ln p_{0} / \partial n$ characterizes the flow vorticity (i.e., its non-isentropic features), and $N_{4}=\delta / y$ expresses the type of flow symmetry ( $N_{4} \equiv 0$ for the plane flow considered here). Also, here $s$ and $n$ are natural coordinates counted along and normally the sreamlines, correspondingly; $M$ is the local Mach number; $p$ is the static pressure; $p_{0}$ is the full (stagnation) pressure of the flow; $y$ is the distance to the axis of symmetry in axis-symmetric flow; $\delta=0$ and $\delta=1$ for the plane flow and for the axis-symmetric one, correspondingly; $\gamma$ is the ratio of specific heats ( $\gamma=1.4$ everywhere if not specifically mentioned otherwise).

The geometrical curvature $K_{\sigma}$ of the shock conventionally remarked here as the additional non-uniformity ( $N_{5} \equiv K_{\sigma}$ ). We admit that the curvature $K_{\sigma}>0$ for the shocks convex downwards (for instance, the shocks $B E$ in Fig. $1 \mathrm{~b}$, and $B C$ in Fig. 1c), and $K_{\sigma}>0$ for shocks convex upwards (e.g., the shock $E C$ in Fig. 1b, and the shock after the point $E$ in Fig. 1a).

\subsection{Prandtl-Meyer Flow Non-uniformities and the Envelope Line of Its Straight Characteristics}

The variation of gas stream parameters at Prandtl-Meyer wave can be described by using the isentropic flow functions. So the wave intensity, i.e., the relation $J_{1}$ of the static pressures behind the wave and after this one:

$$
J_{1}=\pi\left(M_{1}\right) / \pi\left(M_{0}\right)
$$


where, $\pi(M)=\left(1+0.5(\gamma-1) M^{2}\right)^{-\gamma /(\gamma-1)}$ is the isentropic pressure function. The Prandtl-Meyer wave deflection angle is:

$$
\beta_{1}=\chi\left(\omega\left(M_{0}\right)-\omega(M)\right)
$$

Here, $\chi=1$ is the wave direction index, and:

$$
\omega(M)=1 / \sqrt{\varepsilon} \arctan \sqrt{\varepsilon\left(M^{2}-1\right)}-\arctan \sqrt{M^{2}-1}
$$

is the Prandtl-Meyer function, such as:

$$
\begin{gathered}
\frac{d \omega(M)}{d M}=\frac{(1-\varepsilon) \sqrt{M^{2}-1}}{M\left(1+\varepsilon\left(M^{2}-1\right)\right)} \\
\varepsilon=(\gamma-1) /(\gamma+1)
\end{gathered}
$$

Analogously, the strength of the reflected wave $r_{3}$ and flow deflection angle are:

$$
\begin{gathered}
J_{3}=p_{3} / p_{2}=\pi\left(M_{3}\right) / \pi\left(M_{2}\right) \\
\beta_{3}=\chi\left(\omega\left(M_{3}\right)-\omega\left(M_{2}\right)\right)
\end{gathered}
$$

where, $\chi=-1 ; p_{2}, M_{2}, p_{3}$ and $M_{3}$ are pressures and Mach numbers of the flow in regions 2 and 3 (Fig. 1b), consequently.

Later we have to deal with not only the whole waves $r_{1}$ and $r_{3}$ but with their parts the shock wave has just interacted. Such is the sector of the wave $r_{1}$ bounded by the characteristics $O B$ and $E_{1} E$ in Fig. 1b. The flow deflection angle $\beta_{1}^{(\varphi)}$ and the strength $J_{1}^{(\varphi)}$ of this part of the wave are expressed by the formulas:

$$
\begin{aligned}
& \beta_{1}^{(\varphi)}=\chi\left(\omega(M)-\omega\left(M_{1}\right)\right) \\
& J_{1}^{(\varphi)}=\pi(M) / \pi\left(M_{0}\right)
\end{aligned}
$$

Here, $M \equiv M(\varphi)$ is the Mach number at the characteristic $E_{1} E$ determined by the angle $\varphi$ of its inclination:

$$
\varphi=\theta_{0}+\chi\left[\omega\left(M_{0}\right)-\omega(M)+\alpha(M)\right]
$$

where, $\alpha(M)=\arcsin (1 / M)$ is the Mach angle. In a similar manner, the strength of the corresponding sector of the reflected wave $r_{3}$ after the interaction between the shock and the above-mentioned sector of the wave $r_{1}$ is determined as:

$$
J_{3}^{(\varphi)}=\pi\left(M_{3}^{(\varphi)}\right) / \pi\left(M_{2}\right)
$$

The flow deflection angle is

$$
\beta_{3}^{(\varphi)}=\chi\left(\omega\left(M_{3}^{(\varphi)}\right)-\omega\left(M_{2}\right)\right)
$$

where, $\quad \chi=-1$, and $M_{3}^{(\varphi)}$ is Mach number at the last characteristic of the corresponding reflected wave sector. We also designate Mach number of the flow just after the voluntary point of the interacting shock as $\stackrel{M}{M} \equiv \hat{M}(\varphi)$, the flow deflection angle at the interaction shock as $\beta$, and the shock strength (relation of static pressures downstream the shock and upstream it) as $J$.

The Mach lines of the Prandtl-Meyer wave $r_{1}$ have a discriminant curve. This discriminant curve can be a smooth envelope (curve $O_{0} O^{\prime}$ in Figs. 1a and 2a) for a simple (non-centered) wave $r_{1}$. When the part of the straight Mach lines intersects at the single point, the discriminant curve has a sharp bend (Fig. 2b); when all Mach lines have the common point, the Prandtl-Meyer wave becomes centered, and the discriminant curve degenerates into the sole point - the center of either the rarefaction wave (the point $O \equiv O_{0} \equiv E_{1}$ in Fig. 1b) or the compression one. If one part of the Prandtl-Meyer wave realizes the flow rarefaction, and the another part realizes the flow deflection, the envelope line consists of two separate sections (Fig. 2c).

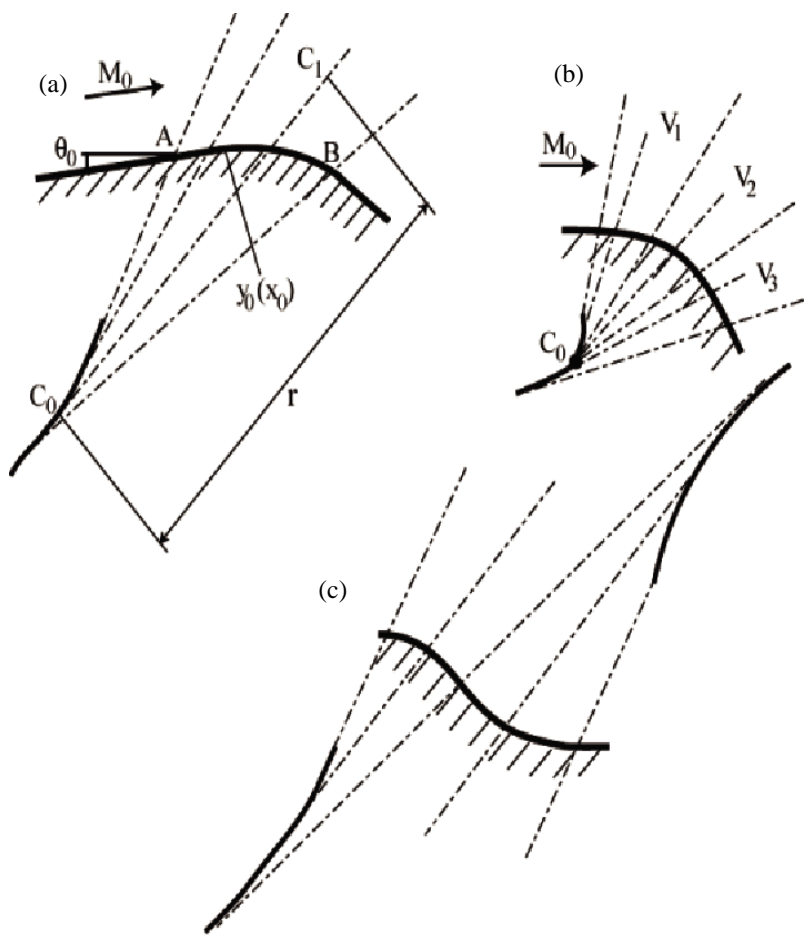

Fig. 2 Discriminant curves of Prandtl-Meyer waves: (a) the smooth envelope of the expansion wave; (b) the expansion wave with a centered sector; (c) the transition of the expansion wave into the compression one and the corresponding discontinuity of the envelope curve. 
The shape of the envelope of the straight Mach lines family is defined by any given streamline of this wave unambiguously. Let the only streamline equation $y_{0}\left(x_{0}\right)$ (for example, the section $A B$ of the impermeable surface in Fig. 2a) to be given, as well as the initial Mach number $M_{0}$ and flow angle $\theta_{0}$. An equation of one-parameter family of the straight characteristics looks like:

$$
y=y_{0}\left(x_{0}\right)+\tan \varphi\left(x_{0}\right) \cdot\left(x-x_{0}\right)
$$

where, $x_{0}$ is the parameter.

The inclination angle $\varphi$ of any straight Mach line and Mach number $M$ on this one are defined by $y_{0}{ }^{\prime}\left(x_{0}\right)$ through Eq. (9) and the following:

$$
y_{0}^{\prime}=\tan \left[\theta_{0}+\chi\left(\omega\left(M_{0}\right)-\omega(M)\right)\right]
$$

The equation $y_{\Gamma}\left(x_{\Gamma}\right)$ for the envelope of the straight family of Mach lines is derived from Eqs. (3), (4), (9), (12) and (13) at the condition $\partial y / \partial x_{0}=0$ [3]:

$$
\begin{gathered}
x_{\Gamma}-x=\frac{\chi(1-\varepsilon)\left(M^{2}-1\right)}{M^{3} N_{2}} \cos \varphi \\
y_{\Gamma}-y=\frac{\chi(1-\varepsilon)\left(M^{2}-1\right)}{M^{3} N_{2}} \sin \varphi \\
r=\frac{(1-\varepsilon)\left(M^{2}-1\right)}{\left|N_{2}\right| M^{3}}
\end{gathered}
$$

Here, $r=\left|C_{0} C_{1}\right|$ is the distance from the voluntary point $C_{1}$ at a straight characteristic to the corresponding point $C_{0}$ at the envelope line. In particular, at $x \equiv x_{0}, y \equiv y_{0}, N_{2}$ is the curvature of the given streamline, and the envelope $y_{\Gamma}\left(x_{\Gamma}\right)$ can be easily built.

The two first basic non-uniformities of the Prandtl-Meyer flow are inversely proportional to the distance $r$ to the envelope along the characteristic line:

$$
\begin{gathered}
N_{1}=\psi \frac{(1+\varepsilon) \sqrt{M^{2}-1}}{r M} \\
N_{2}=\chi \psi \frac{(1-\varepsilon)\left(M^{2}-1\right)}{r M^{3}}
\end{gathered}
$$

(here $\psi=1$ for the compression wave, and $\psi=-1$ for the expansion one).

The spatial derivative of any variable flow parameter $f$ given in the voluntary direction $\tilde{q}$ inside Prandtl-Meyer wave depends on the angle between $\tilde{q}$ and gradient vector grad $f$ which is normal to the straight characteristics:

$$
\partial f / \partial q=\stackrel{\mu}{q} \cdot \operatorname{grad} f
$$

The Eqs. (9), (13) and (16), and the well-known functions of the isentropic flow allow us to calculate all flow parameters derivatives along the direction of the oblique shock coming through Prandtl-Meyer fan of characteristics situated upstream.

\subsection{Dynamic Compatibility Conditions at Stationary Shocks}

Among the numerous variables for shock description, we choose the shock strength $J$ (i.e., the relation of the static pressures downstream and upstream the shock) as the main parameter. We denote below that $J$ is the strength of the shock inside the Prandtl-Meyer fan, $J_{2}=p_{2} / p_{1}$ is the strength of the shock $j_{2}$, and $J_{4}$ is the strength of the resulting shock $j_{4}$. Here, $p_{1}$ is the pressure in zone 1 , and $p_{2}$ is the pressure in zone 2 of the flow (Fig. 1).

The shock strength here is the main quantity binding flow parameters before and behind the shock. For example, Mach numbers $M_{2}$ (downstream the shock $j_{2}$ ) and $M_{1}$ (upstream the shock) relate between themselves as it follows:

$$
M_{2}=\sqrt{\frac{\left(J_{2}+\varepsilon\right) M_{1}^{2}-(1-\varepsilon)\left(J_{2}^{2}-1\right)}{J_{2}\left(1+\varepsilon J_{2}\right)}}
$$

The flow deflection angle $\beta_{2}$ also depends upon the shock strength:

$$
\beta_{2}=\arctan \left[\sqrt{\frac{J_{m}\left(M_{1}\right)-J_{2}}{J_{2}+\varepsilon}} \frac{(1-\varepsilon)\left(J_{2}-1\right)}{J_{m}\left(M_{1}\right)+\varepsilon-(1-\varepsilon)\left(J_{2}-1\right)}\right]
$$

Here, $J_{m}(M)=(1+\varepsilon) M^{2}-\varepsilon$ is the strength of the normal shock in the flow with Mach number $M$. The relations analogous to Eqs. (17) and (18) are correct for the shock $j_{4}$ :

$$
M_{4}=\sqrt{\frac{\left(J_{4}+\varepsilon\right) M_{0}^{2}-(1-\varepsilon)\left(J_{4}^{2}-1\right)}{J_{4}\left(1+\varepsilon J_{4}\right)}}
$$




$$
\beta_{4}=\arctan \left[\sqrt{\frac{J_{m 0}-J_{4}}{J_{4}+\varepsilon}} \frac{(1-\varepsilon)\left(J_{4}-1\right)}{J_{m 0}+\varepsilon-(1-\varepsilon)\left(J_{4}-1\right)}\right]
$$

(here, $J_{m 0} \equiv J_{m}\left(M_{0}\right)$ ) and also for any point at the interacting part $B C$ of the shock considered:

$$
\begin{gathered}
M=\sqrt{\frac{(J+\varepsilon) M^{2}-(1-\varepsilon)\left(J^{2}-1\right)}{J(1+\varepsilon J)}} \\
\beta=\arctan \left[\sqrt{\frac{J_{m}(M)-J}{J+\varepsilon}} \frac{(1-\varepsilon)(J-1)}{(1+\varepsilon) M^{2}-(1-\varepsilon)(J-1)}\right]
\end{gathered}
$$

The angle $\sigma$ of shock inclination relative to the flow velocity vector upstream the given point is also bound with shock strength $(J)$ and local Mach number upstream $(M)$ :

$$
J=(1+\varepsilon) M^{2} \sin ^{2} \sigma-\varepsilon
$$

In the coordinate system admitted here, Eq. (23) determines the dependence of shock slope angle $\xi$ (Fig. 1) on the shock strength:

$$
\xi=\sigma+\beta_{1}^{(\varphi)}
$$

that is an equivalent to the quantity $y_{\sigma}{ }^{\prime}\left(x_{\sigma}\right)$ where $y_{\sigma}\left(x_{\sigma}\right)$ is the shock shape looked for.

Except of $J_{m}(M)$, we must emphasize here the following special shock strengths: the strength of the shock declining the flow to a maximum angle possible at a given Mach number:

$$
J_{l}(M)=\frac{M^{2}-2}{2}+\sqrt{\left(\frac{M^{2}-2}{2}\right)^{2}+(1+2 \varepsilon)\left(M^{2}-1\right)+2}
$$

and the strength of the shock reducing the flow velocity downstream it to the critical speed:

$$
J_{*}(M)=\frac{M^{2}-1}{2}+\sqrt{\left(\frac{M^{2}-1}{2}\right)^{2}+\varepsilon\left(M^{2}-1\right)+1}
$$

Dependencies $J_{*}(M), J_{l}(M)$ and $J_{m}(M)$ are given in Fig. 3 (curves 1, 2 and 3, consequently). At all Mach numbers $M>1$, the inequality $1<J_{*}(M)<J_{l}(M)<J_{m}(M)$ is correct.

Eqs. (17)-(22) and a lot of similar others express zero-order dynamic compatibility conditions (i.e., they bind flow parameters, not their spatial derivatives). To connect the spatial derivatives of the flow parameters downstream and upstream the shock, we should use first-order (differential) dynamic compatibility conditions.

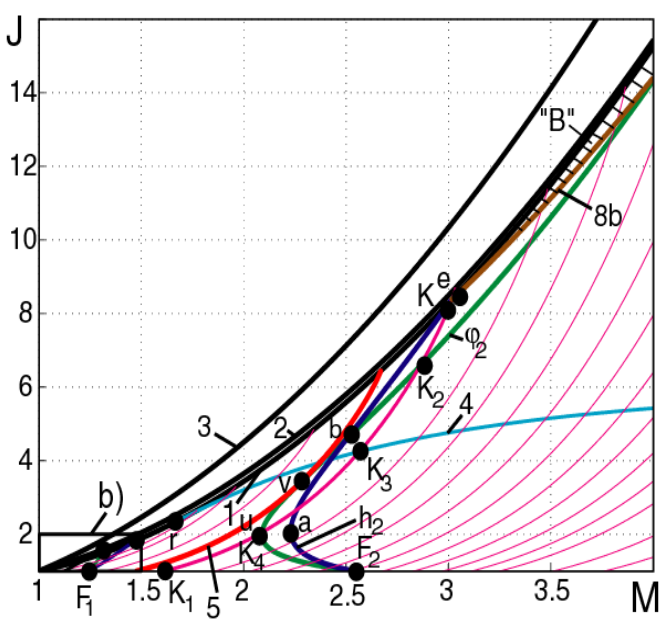

(a)

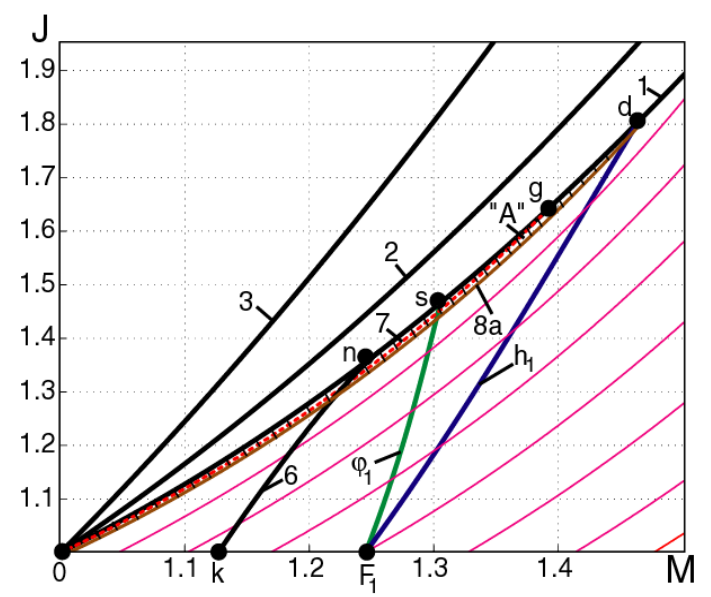

(b)

Fig. 3 The parametric solution of the interaction problem considered: (a) general solution; (b) the region of small Mach numbers.

Several forms of that unambiguous conditions have been published already (e.g., in Refs. [4, 5]; the universal form for strong discontinuities in the solution of two-variables quasi-linear system is given in Ref. [6]).

To express the correlation between the basic non-uniformities downstream ( $\left.\dot{N}_{j}, j=1 \ldots 3\right)$ and upstream $\left(N_{i}, i=1 \ldots 5\right)$ the shock with the strength $J$ occurred in the flow with Mach number $M$, we use the differential dynamic compatibility conditions in the following form [2]:

$$
\hat{N}_{j}=C_{j} \sum_{i=1}^{5} A_{j i} N_{i}
$$

where, $C_{j}$ and $A_{j i}$ are factors depending on $M, J$, 
shock direction $\chi$ ( $\chi=1$ everywhere at the present study), and the flow angle $\theta$ (only at axis-symmetric flow). The factors we are interested in look as:

$$
\begin{aligned}
& C_{1}=\gamma /(\sqrt{b}(J-1)), \\
& C_{2}=(1-\varepsilon) J /\left(\sqrt{b^{3}}(J-1)\right), \\
& A_{11}=-\frac{1}{\gamma} \sqrt{\frac{J+\varepsilon}{\left(J_{m}+\varepsilon\right)^{3}}}\left[a_{p}\left(J_{m}-J\right)^{2}-b_{p}\left(J_{m}-J\right)+c_{p}\right], \\
& A_{12}=-\frac{\chi S}{1+\varepsilon}\left[a_{\theta}(J+\varepsilon)^{2}+b_{\theta}(J+\varepsilon)+c_{\theta}\right], \\
& A_{15}=-\chi(1-\varepsilon)\left(a_{\omega} J^{2}-b_{\omega} J-c_{\omega}\right), \\
& A_{21}=\frac{\chi s}{J}\left[f_{1}+\left(J_{m}-1\right) c^{2} f_{2} / \gamma\right], \\
& A_{22}=-\frac{c}{J}\left[\frac{J_{m}+\varepsilon}{1-\varepsilon} f_{1}+\left(J_{m}-J\right) f_{2}\right], \\
& A_{25}=a / J \\
& \cdot\left\{2 m+J(1+\varepsilon)\left[\left(J_{m}-J\right)(J+1+2 \varepsilon)-(1+\varepsilon J)(J-1)\right]\right\}
\end{aligned}
$$

where:

$$
\begin{aligned}
& a=\left[\left(J_{m}-J\right)(J+\varepsilon)\right]^{1 / 2}, \\
& b=a^{2}+(1+\varepsilon J)^{2}, \\
& c=\left[(J+\varepsilon) /\left(J_{m}+\varepsilon\right)\right]^{1 / 2}, \\
& s=\left[\left(J_{m}-J\right) /\left(J_{m}+\varepsilon\right)\right]^{1 / 2}, \\
& a_{p}=(1-\varepsilon)\left(3 J_{m}-4-\varepsilon\right), \\
& b_{p}=(3-2 \varepsilon) J_{m}^{2}-2\left(1-\varepsilon+3 \varepsilon^{2}\right) J_{m}-\left(4+6 \varepsilon-3 \varepsilon^{2}\right), \\
& c_{p}=\left(J_{m}-1\right)\left(1+\varepsilon J_{m}\right)\left(J_{m}+\varepsilon\right), \\
& a_{\Theta}=(1-\varepsilon)\left[J_{m}+\varepsilon-4(1+\varepsilon)\right], \\
& b_{\Theta}=2(1+\varepsilon)\left((1-3 \varepsilon)\left(J_{m}+\varepsilon\right)-2\left(1-\varepsilon^{2}\right)\right), \\
& c_{\Theta}=(1+\varepsilon)^{2}(1-\varepsilon)\left(J_{m}+\varepsilon\right), \\
& a_{w}=3+\varepsilon, \\
& b_{w}=3 J_{m}-2-3 \varepsilon, \\
& c_{w}=(1+4 \varepsilon) J_{m}+1 ; \\
& f_{1}=m J+2 \mu(J+\varepsilon)\left(m+(1+\varepsilon)^{2}\left(J_{m}-J\right) J\right), \\
& f_{2}=2 m-\gamma J\left(\left(1+\varepsilon J_{m}\right)(J+\varepsilon)-\left(1-\varepsilon^{2}\right)\left(J_{m}-J\right)\right), \\
& m=(1+\varepsilon J)[b-(1+\varepsilon) J(1+\varepsilon J)], \\
& \mu=-\left(1+\varepsilon J_{m}\right)\left[(1+\varepsilon)\left(J_{m}+\varepsilon\right)\right]^{-1} .
\end{aligned}
$$

To define the flow non-uniformities downstream the shock, as seen from Eq. (25), it is enough to know the geometrical curvature of the shock $\left(N_{5} \equiv K_{\sigma}\right)$.

\subsection{Interaction of the Oblique Shock with Preceding} Overtaking Weak Discontinuities

The last $(O B)$ and the first $\left(O_{0} C\right.$, Figs. 1b-1d) straight characteristics of the wave $r_{1}$ are the weak discontinuities, i.e., the discontinuities of the basic non-uniformities $N_{1}$ and $N_{2}$. Since these non-uniformities are equal to zero in zone 0 and correspond to Eqs. (14) and (15) inside Prandtl-Mayer wave, we can determine the discontinuities $\left[N_{1}\right]$ and $\left[N_{2}\right]$ at the characteristic line $O_{0} C$ :

$$
\begin{aligned}
& {\left[N_{1}\right]=\psi \frac{(1+\varepsilon) \sqrt{M^{2}-1}}{r M}} \\
& {\left[N_{2}\right]=\psi \frac{(1-\varepsilon)\left(M^{2}-1\right)}{r M^{3}}}
\end{aligned}
$$

On the contrary, the flow is non-uniform (also corresponds to Eqs. (14) and (15)) before the Mach line $O B$ and uniform behind it. So the discontinuities of the basic non-uniformities at the last characteristic of the Prandtl-Meyer wave are:

$$
\begin{aligned}
& {\left[N_{1}\right]=-\psi \frac{(1+\varepsilon) \sqrt{M^{2}-1}}{r M}} \\
& {\left[N_{2}\right]=-\psi \frac{(1-\varepsilon)\left(M^{2}-1\right)}{r M^{3}}}
\end{aligned}
$$

The distance $r$ for centered or non-centered wave, as it was shown in Subsection 2.2, can be easily determined owing to the given equation of the streamlined surface.

It is worthy of notice from Eqs. (26)-(29) that the relation:

$$
\left[N_{1}\right]-\chi \Gamma(M)\left[N_{2}\right]=0
$$

expresses the dynamical conditions of compatibility at weak discontinuities of gas flow parameters (here $\Gamma(M)=\gamma M^{2} / \sqrt{M^{2}-1} ; \chi=1$ for wave $r_{1}$ with the straight characteristics of the first family).

The problem of the interaction of the stationary shock with the preceding overtaking weak discontinuity has the following solution [2] in variables accepted here: 


$$
\frac{\left[K_{\sigma}\right]}{\left[N_{2}\right]}=\frac{C_{1}\left(\Gamma A_{11}+A_{12}\right)+C_{2} \stackrel{\Gamma}{\Gamma}\left(\Gamma A_{21}+A_{22}\right)}{C_{1} A_{15}+C_{2} \Gamma A_{25}}
$$

Here, $\Gamma \equiv \Gamma(M), \quad \dot{\Gamma} \equiv \Gamma\left(M^{\prime}\right), \quad M$ is the Mach number upstream the point of the intersection, and $M^{\prime}$ is the Mach number behind the shock at this point (for example, $M=M_{1}$ and $M^{\prime}=M_{2}$ at point $B$ ).

Eqs. (27), (29) and (30) allow us not only to define the discontinuities of shock curvature at points $B$ and $C$, but also to determine the shock curvature just after the point $B$ quite exactly ( $K_{\sigma}=\left[K_{\sigma}\right]$, Eq. (29)). Declaring that the curvature of the shock $j_{4}$ is equal to zero right after the point $C$, we can determine the shock curvature of the interacting shock $B C$ just before this point ( $K_{\sigma}=-\left[K_{\sigma}\right]$, Eq. (27)). But, as it was shown in Section 1, this assumption is not absolutely exact and needs some proof for its introduction.

\subsection{Problem Solution Based on the Assumption of Zero} Curvature of the Resulting Shock

The calculations carried out by the second-order method of characteristics at utmost asymptotic refining of the numerical grid have shown that the shock $j_{4}$ is of an extremely small curvature (in particular, at point $C$ ). For example, in case of the interaction with the centered expansion fan, its curvature is equal to $2 \cdot 10^{-6}$ at $M_{1}=1.5, J_{1}=0.8$ and $J_{2}=1.4$; to $3 \cdot 10^{-5}$ at $M_{1}=3, J_{1}=0.4$ and $J_{2}=4$; to $4 \cdot 10^{-6}$ at $M_{1}=5, J_{1}=0.3$ and $J_{2}=10$. The intersection with the weak discontinuity $O_{0} C$ diminishes the shock geometric curvature approximately by 1,200 times in the first case, by 800 times in the second case, and by 4,500 times in the third one. So we can conclude that the curvature of the shock $j_{4}$ is very small compared with the curvature of the interacting shock $B C$ and can be accepted to be equal to zero. Then the curvature of the shock $B C$ at point $C$ can be easily determined from Eqs. (27) and (30): $K_{\sigma}=-\left[K_{\sigma}\right]$.

Let us now consider the shock which has interacted not with the whole expansion fan, but with its part bounded by the straight characteristics $O B$ and $E_{1} E$ (Figs. 1a and $1 \mathrm{~b}$ ). The following interaction of this shock with the remaining part of the wave does not change the shock section $E C$. So the assumption of zero shock curvature at the point of its exit out of the Prandtl-Meyer fan is equivalent to the possibility of using the relation analogous to Eq. (30) at all inner points of the interacting shock:

$$
K_{\sigma}=\frac{C_{1}\left(\Gamma A_{11}+A_{12}\right)+C_{2} \zeta\left(\Gamma A_{21}+A_{22}\right)}{C_{1} A_{15}+C_{2} \Gamma A_{25}} \cdot N_{2}
$$

Considering Eqs. (15), (21) and (31), we conclude that the dimensionless curvature of the interacting shock depends only on wave type $\psi= \pm 1$, the shock strength $J$ and the local Mach number $M$ upstream:

$$
\begin{aligned}
r K_{\sigma}=\psi & \cdot \frac{C_{1}\left(\Gamma A_{11}+A_{12}\right)+C_{2} \sum\left(\Gamma A_{21}+A_{22}\right)}{C_{1} A_{15}+C_{2} \Gamma A_{25}} \times \\
& \times \frac{(1-\varepsilon)\left(M^{2}-1\right)}{M^{3}} .
\end{aligned}
$$

Since $K_{\sigma}=y_{\sigma}^{\prime \prime} /\left(1+\left(y_{\sigma}^{\prime}\right)^{2}\right)^{3 / 2}$, where, $y_{\sigma}\left(x_{\sigma}\right)$ is the shape of the shock, Eq. (31) allows us to determine the shape of the shock in the interaction region. Eqs. (17)-(23) binding shock shape and strength and Eqs. (2)-(4), (9) and (12)-(16) which allow us to calculate flow parameters derivatives at all points and in all directions inside the Prandtl-Meyer fan lead to the following equations complementary to Eq. (31):

$$
\begin{gathered}
d J / d M=2(J+\varepsilon) f_{3} / M, \\
f_{3}=1+\frac{(1-\varepsilon) \sqrt{M^{2}-1}}{1+\varepsilon\left(M^{2}-1\right)} \cdot \sqrt{\frac{J_{m}(M)-J}{J+\varepsilon}} \cdot f_{4}, \\
f_{4}=1-\frac{C_{1}\left(\Gamma A_{11}+A_{12}\right)+C_{2} \Gamma\left(\Gamma A_{21}+A_{22}\right)}{M \sin (\sigma-\alpha) \cdot\left(C_{1} A_{15}+C_{2} \Gamma A_{25}\right)},
\end{gathered}
$$

where, to exclude trigonometric expressions,

$$
\sin (\sigma-\alpha)=\frac{\sqrt{(J+\varepsilon)\left(M^{2}-1\right)}-\sqrt{J_{m}(M)-J}}{M^{2} \sqrt{1+\varepsilon}}
$$

(the intermediate calculations are contained in Ref. [7]).

Taking into account that the derivative $d J / d M$ does not depend on upstream flow non-uniformities, we must conclude that Eq. (32) is most convenient to solve the problem. Neither initial data $\left(M_{1}, J_{2}\right.$, etc.) nor local non-uniformities, but only local Mach number $M$ and shock strength $J$ participate in this 
equation. The integral curves of Eq. (32) are shown in Figs. 3a and 3b as slim lines. The shock strength decrease under the influence of the rarefaction wave corresponds to the motion downward these curves; the increase of the strength $J$ at the interaction with the compression wave corresponds to the motion upward. Mach number at sonic line 1 ( $J=J_{*}(M)$ there) or on abscissa axis ( $J=1$; that corresponds to the shock degeneration) can be considered as the only parameter.

In fact, the assumption of zero curvature of the resulted shock implied the neglect to the influence of the perturbations refracted at the entropy layer on the shock parameters. These assumptions concerning the moving shock waves in non-steady flow underlie Chester, Chisnell and Whitham's approximate analytical methods [8]. Analogous assumptions concerning shocks in steady flow were introduced in Ref. [9] and other studies.

\subsection{The Solution Based on the Static Pressure Equality} and Collinearity of Flow Directions Downstream the Resulting Waves

The straightness of the shock $J_{4}$ in Fig. 1 is possible only at the absence of the perturbations which overtakes the shock from behind. The equality of static pressures and the collinearity of the streams on both sides of vortex layer 5 is a necessary (not sufficient, though) condition for it. These conditions can be formulated as the system binding wave strengths $J_{i}$ and deflection angles $\beta_{i}(i=1 \ldots 4)$ :

$$
\begin{aligned}
& \quad J_{1} J_{2} J_{3}=J_{4} \\
& \beta_{1}\left(M_{0}, J_{1}\right)+\beta_{2}\left(M_{1}, J_{2}\right)+\beta_{3}\left(M_{2}, J_{3}\right)= \\
& =\beta_{4}\left(M_{0}, J_{4}\right)
\end{aligned}
$$

Shock strengths $J_{2}$ and $J_{4}$, corresponding deflection angles, and Mach numbers are tied together with Eqs. (17)-(20), and the same flow variables upstream and downstream Prandtl-Meyer waves are bound with Eqs. (1), (2), (5) and (6).

Using Eq. (33) in logarithmic form $\left(\Lambda_{i}=\ln J_{i}\right)$, it is not difficult to solve the system Eqs. (33) and (34) owing to the well-known technique of shock and expansion-compression polars.

Like Eq. (30) in the previous model, Eqs. (33) and (34) can be generalized for the inner points of the interacting curvilinear shock:

$$
\begin{gathered}
J_{1}^{(\varphi)} J_{2} J_{3}^{(\varphi)}=J \\
\beta_{1}^{(\varphi)}\left(M(\varphi), J_{1}^{(\varphi)}\right)+\beta_{2}\left(M_{1}, J_{2}\right)+ \\
+\beta_{3}^{(\varphi)}\left(M_{2}, J_{3}^{(\varphi)}\right)=\beta(M(\varphi), J)
\end{gathered}
$$

Here, $M(\varphi)$ is Mach number at a straight characteristic coming into the given point of the shock; $J_{1}^{(\varphi)}=\pi\left(M_{1}\right) / \pi(M(\varphi))$ is the strength of the Prandtl-Meyer wave part which have just interacted with the shock; $J_{3}^{(\varphi)}=\pi\left(M_{3}^{(\varphi)}\right) / \pi\left(M_{2}\right)$ is the strength of the corresponding part of the reflected wave $r_{3}$; $M_{3}^{(\varphi)}$ is the Mach number at the last characteristic of this reflected part; $\beta_{1}^{(\varphi)}$ and $\beta_{3}^{(\varphi)}$ are the flow deflection angles at the named parts of the waves $r_{1}$ and $r_{3}$. Shock strengths $J_{2}$ and $J$, corresponding deflection angles, and the Mach numbers are tied here by Eqs. (17), (18), (21) and (22). Corresponding flow parameters at Prandtl-Meyer wave sectors are bound by Eqs. (7), (8), (10) and (11).

The system Eqs. (35) and (36) determines the local shock strength $J$ and deflection angle $\beta$ in any inner point of the interacting shock.

Differentiating Eqs. (35) and (36), we can get the expressions analogous to Eq. (31) for the shock curvature, and the expressions analogous to Eq. (32) for the shock strength variation. But, unlike in Eqs. (31) and (32), the initial parameters ( $M_{1}$ and $J_{2}$ ) are present in these expressions which become rather more complicated [7]. So the solutions of Eqs. (33) and (34) do not compose the one-parameter family of curves at the $(M, J)$-plane. The system Eqs. (33) and (34) is definitely not an integral of Eq. (32).

Conditions Eqs. (33) and (34) were, in fact, introduced in Ref. [10] to solve the problem of the interaction between the shock and Prandtl-Meyer wave of opposite direction [11]. Eqs. (33)-(36) are formally analogous to the compatibility conditions in the case of two overtaking shocks intersections. But Eqs. (33) and 
(34) give us, as a rule, only an approximate solution of the problem because we neglect the influence of refracted perturbations on flow downstream the shock $j_{4}$ and the wave $r_{3}$.

\section{Results and Discussion}

\subsection{The Accuracy of the Methods Proposed}

The results achieved due to the "differential" model Eqs. (31) and (32) and the "integral" one Eqs. (35) and (36) demonstrate a very high accuracy.

The errors $\Delta J=J-J_{e}$ of the shock strength computation resulting at the application of Eqs. (31) and (32) and Eqs. (35) and (36) are shown in Fig. 4 relative to the series of incoming centered expansion wave strengths $J_{1}$ given shock interacts with. Here, $J_{e}$ 's are the strengths of the interacting shock at the same $M_{1}$, $J_{2}$ and $J_{1}$ 's calculated by second-order method of characteristics with asymptotic grid refining and accepted here as being exact. Curves 1 in Fig. 4 show us the errors of the model based on Eqs. (31) and (32), and curves 2 —of the model given by Eqs. (35) and (36).

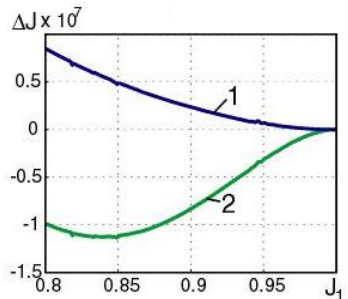

(a)

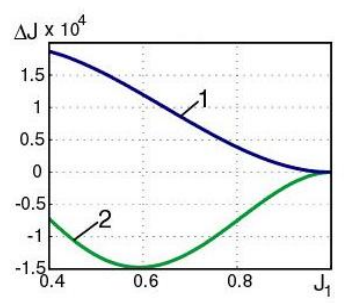

(c)

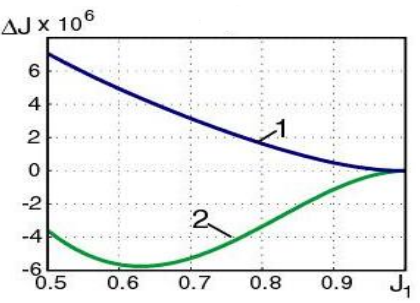

(b)

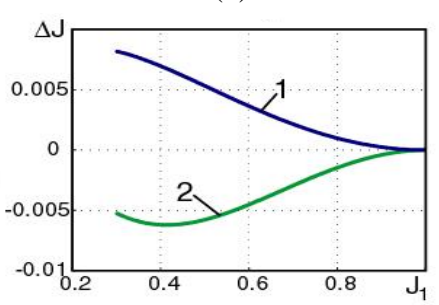

(d)
Fig. 4. The errors in the approximate analytical solutions Eqs. (31) and (32) and Eqs. (35) and (36) at the interaction of a given shock with a series of the centered expansion waves: (a) $M_{1}=1.5, J_{2}=1.4, J_{1}=0.8 \ldots 1.0$; (b) $M_{1}=2, J_{2}=2.5, J_{1}=0.5 \ldots 1.0 ;$ (c) $M_{1}=3$, $J_{2}=4, \quad J_{1}=0.4 \ldots 1.0 ;$ (d) $M_{1}=5, J_{2}=10$, $J_{1}=0.3 \ldots 1.0$.
The errors in the proposed models are small enough relatively to both the shock strength total variation $\left(J_{2}-J_{4}\right)$ and the pressure variation in the reflected wave $r_{3}$. For example, $J_{3}=1.014$ at $M_{1}=3, J_{1}=0.4$ and $J_{2}=4$, and the errors of the resulting shock strength according to the above-mentioned models are $\Delta J_{4}=1.8 \cdot 10^{-4}$ and $\Delta J_{4}=7 \cdot 10^{-5}$, correspondingly.

The model [1] based on the neglect to the reflected waves at all $\left(J_{3} \equiv 1\right)$ is qualitatively less accurate ( $\Delta J \approx 0.02 \div 0.4$ for the cases shown in Figs. $4 \mathrm{a}-4 \mathrm{~d}$ ).

So, owing to very high order of smallness of the neglected perturbations reflected from the vortex layer and influencing the shock from behind, the solutions proposed here are accurate enough to study the type of the reflected wave $r_{3}$ and other special features of the interaction considered.

\subsection{The Inflections of the Interacting Shock}

The relation Eq. (31) determines the geometric curvature of the interacting shock in an explicit form though this formula can not be applied to the shock with the subsonic flow downstream. In the critical case ( $J=J_{*}(M), M^{\prime} \rightarrow 1$ ) the shock curvature is finite, and Eq. (31) with the coefficients $A_{2 i}$ computed at $J=J_{*}(M)$ confirms it.

As it follows from Eq. (31), rather strong shocks interacting with the expansion wave are convex downwards (for example, the section $B E$ of the shock in Fig. 1b). The loss of the shock strength under the further influence of the expansion wave leads to shock inflection, and the curvature becomes negative (such is the section $E C$ ). The negative curvature of the shock degenerating inside the expansion fan into a weak discontinuity as shown in Fig. 1a asymptotically strives to zero. If the shock interacts with the compression wave, the sign of its curvature changes in a reverse order.

The location of the shock inflection point is determined by Eq. (31) at the condition $K_{\sigma}=0$. The shock curvature direction changes when shock parameters cross curve 4 in Fig. 3a. Curve 4 goes out 
the point $r$ at the "critical" ("sonic") line 1. The finite limit of the shock curvature determined from Eq. (31) at $M^{\prime} \rightarrow 1\left(J \rightarrow J_{*}(M)\right)$ :

$$
K_{\sigma}=\frac{\Gamma A_{21}\left(M, J_{*}\right)+A_{22}\left(M, J_{*}\right)}{A_{25}\left(M, J_{*}\right)} \cdot N_{2}
$$

leads to the equations

$$
(1-\varepsilon) M_{r}^{6}-(5-9 \varepsilon) M_{r}^{4}+12(1-2 \varepsilon) M_{r}^{2}-16(1-\varepsilon)=0
$$

for Mach number $M_{r}=1.676$ and

$J_{r}^{3}-2 J_{r}^{2}-3 \varepsilon J_{r}-(1-\varepsilon)=0$

for the shock strength $J_{r}=J_{*}\left(M_{r}\right)=2.361$ at the point $r$ where the inflection line starts. Inflection line 4 has the horizontal asymptote $\left(J=J_{A}=6.360\right)$ at $M \rightarrow \infty$ where, $J_{A}$ is the root of the following equation:

$\sum_{i=0}^{6} B_{i} J_{A}^{i}=0$,

$B_{6}=\varepsilon^{2}(1-\varepsilon)^{4}$,

$B_{5}=2 \varepsilon(1-\varepsilon)^{2}\left(1-5 \varepsilon+3 \varepsilon^{2}-3 \varepsilon^{3}\right)$,

$B_{4}=1-16 \varepsilon+47 \varepsilon^{2}-48 \varepsilon^{3}+71 \varepsilon^{4}-$

$-64 \varepsilon^{5}+9 \varepsilon^{6}+16 \varepsilon^{7}$,

$B_{3}=-6+26 \varepsilon-36 \varepsilon^{2}+100 \varepsilon^{3}-158 \varepsilon^{4}-30 \varepsilon^{5}-$

$-72 \varepsilon^{6}-16 \varepsilon^{7}+16 \varepsilon^{8}$,

$B_{2}=(1-2 \varepsilon)$.

$\cdot\left(1-10 \varepsilon+42 \varepsilon^{2}+40 \varepsilon^{3}+161 \varepsilon^{4}+122 \varepsilon^{5}+44 \varepsilon^{6}+16 \varepsilon^{7}\right)$,

$B_{1}=-8 \varepsilon^{2}\left(1-7 \varepsilon+6 \varepsilon^{2}-\varepsilon^{3}+7 \varepsilon^{4}+14 \varepsilon^{5}+4 \varepsilon^{6}\right)$,

$B_{0}=16 \varepsilon^{4}\left(1-\varepsilon-\varepsilon^{2}\right)^{2}$.

When we try to determine the shock curvature from Eqs. (35) and (36), the shock inflection depends not only on the local parameters ( $M$ and $J$ ) but also on the initial data for the incoming shock ( $M_{1}$ and $J_{2}$ ).

But the results of the applications of the two proposed models can be barely distinguished. So for $M_{1}=3$ and $J_{2}=6$ the shock inflection occurs at $M=2.808$ and $J=4.557$ according to Eq. (31). Determining the shock curvature from Eqs. (35) and (36), it was calculated that the inflection point corresponds to $M=2.806$ and $J=4.556$.

\subsection{The Reflected Wave and the Change of Its Type}

Change of flow types (expansion or compression) in the wave $r_{3}$ can be analyzed based on Eqs. (35) and (36). The second-family characteristic where the wave $r_{3}$ changes its type corresponds to the extremum of current strength $J_{3}^{(\varphi)}$ of the reflected wave part:

$$
J_{3}^{(\varphi)}\left(J_{1}^{(\varphi)}\right) \rightarrow \text { extr }
$$

with the limitations Eqs. (35) and (36) binding $J_{1}^{(\varphi)}$ and $J_{3}^{(\varphi)}$ in an implicit form. The solution of Eq. (37) depends only on the local Mach number $M$ and the shock strength $J$ :

$$
\sum_{i=0}^{3} D_{i} M^{2 i}=0
$$

$D_{3}=J^{2}(1+\varepsilon)^{2}-4 \varepsilon(J+\varepsilon)^{2}$,

$D_{2}=4 \varepsilon(1-\varepsilon)(J+\varepsilon)\left(J^{2}-1\right)-$

$-2\left(1-\varepsilon^{2}\right) J^{2}(J-1)-4(1-2 \varepsilon)(J+\varepsilon)^{2}$,

$D_{1}=(1-\varepsilon)$.

$\cdot\left[4(1-2 \varepsilon)\left(J^{2}-1\right)(J+\varepsilon)+4(J+\varepsilon)^{2}+(1-\varepsilon) J^{2}(J-1)^{2}\right]$,

$D_{0}=-4(1-\varepsilon)^{2}(J+\varepsilon)\left(J^{2}-1\right)$.

Eq. (38) determines the curves $\varphi_{i}(i=1,2)$ at $(M, J)$-plane (Figs. 3a and $3 \mathrm{~b})$. If $r_{1}$ is the expansion Prandtl-Meyer flow then the regions I and II correspond to the reflection of compression waves, and the region III to the reflection of rarefaction waves. The contrary conclusions are correct if $r_{1}$ is the compression wave.

Curves $\varphi_{i}$ emerge from the points $F_{i}$ at the axis $J=1$. The corresponding Mach numbers $\left(M_{F_{1}}=1.245\right.$, $M_{F_{2}}=2.540$ ) are determined by the relation:

$$
M_{F_{1,2}}=\sqrt{2(1 \pm \sqrt{\varepsilon}) /(1 \pm 2 \sqrt{\varepsilon})}
$$

These special Mach numbers were $\mathrm{d}$ discovered earlier in the problems of overtaking shock-shock anshock-weak discontinuity interactions. They occur also in the study of the systems which consist of Prandtl-Meyer waves and subsequent overtaking shocks and provide extreme static pressure at fixed total flow deflection [12, 13].

The curve $\varphi_{1}$ reaches the "sonic" line 1 at the point $s \quad\left(M_{s}=1.305, J_{s}=J_{*}\left(M_{s}\right)=1.466\right)$ with the coordinates determined by the equations: 


$$
\begin{aligned}
& 4 \varepsilon M_{s}^{6}+\left(3+2 \varepsilon-9 \varepsilon^{2}\right) M_{s}^{4}+ \\
& +4\left(1-8 \varepsilon+6 \varepsilon^{2}\right) M_{s}^{2}-16(1-\varepsilon)^{2}=0, \\
& 4 \varepsilon J_{s}^{3}+3(1-\varepsilon)^{2} J_{s}^{2}-\left(5-2 \varepsilon+5 \varepsilon^{2}\right) J_{s}+ \\
& +\left(1-3 \varepsilon-\varepsilon^{2}-\varepsilon^{3}\right)=0 .
\end{aligned}
$$

The coordinates $\left(M_{u}=2.089, J_{u}=1.989\right)$ of the point $u$ where the curve $\varphi_{2}$ has a vertical tangent are determined by more complex expressions (for example, $J_{u}(\varepsilon)$ can be presented as a root of an eighth-order algebraic equation). To find out its coordinates it is easier to solve the system which consists Eq. (38) and the following equation:

$$
\begin{aligned}
& \sum_{i=0}^{3} E_{i} M_{u}^{2 i}=0, \\
& E_{3}=2(1-\varepsilon)^{2} J-8 \varepsilon^{2}, \\
& E_{2}=-2\left(3(1-\varepsilon)^{2} J^{2}+2\left(1-4 \varepsilon-\varepsilon^{2}+2 \varepsilon^{3}\right) J+2 \varepsilon(3-5 \varepsilon)\right), \\
& E_{1}=2(1-\varepsilon) . \\
& \cdot\left[2(1-\varepsilon) J^{3}+3(1-3 \varepsilon) J^{2}+(1-\varepsilon)(5+8 \varepsilon) J-2(1-4 \varepsilon)\right], \\
& E_{0}=4(1-\varepsilon)^{2}\left(1-2 \varepsilon J-3 J^{2}\right) .
\end{aligned}
$$

As it is seen in Fig. 3a, one of the integral curves of Eq. (32) has point $v$ of contact with the curve $\varphi_{2}$. To achieve its coordinates $\left(M_{v}=2.282, J_{v}=3.434\right.$ ), we have to equate the slopes of the curve $\varphi_{2}$ and the integral curve of Eq. (32). The thick integral curve 5 of Eq. (32) descending from the point ( $M=2.670$, $\left.J=J_{*}(M)=6.440\right)$ at the "sonic" line 1 to the point ( $M=1.477, J=1$ ) contacted the curve $\varphi_{2}$ at the point $v$. All the integral curves situated to the right of curve 5 correspond to at least one reflected wave type change point.

The curve $\varphi_{2}$ asymptotically reaches the line $J=M^{2}-1$ at $M \rightarrow \infty$.

Using the "differential” curved shock model Eqs. (31) or (32) and differential conditions (25) of the dynamic compatibility at the interacting shock as well as Eqs. (14) and (15) we went to the following correlation of flow non-uniformities upstream and downstream the shock:

$$
\begin{aligned}
& \frac{\hat{N}_{1}}{N_{1}}=\frac{\dot{N}_{2}}{N_{2}}= \\
& =-\frac{C_{1} C_{2}\left[A_{15}\left(\Gamma A_{21}+A_{22}\right)-A_{25}\left(\Gamma A_{11}+A_{12}\right)\right]}{C_{1} A_{15}+C_{2} \Gamma A_{25}}
\end{aligned}
$$

We have to note the following correlation of the basic non-uniformities immediately after every inner point of the curved shock:

$$
\hat{N}_{1}+\Gamma N_{2}=0
$$

which is analogous to the relation correct everywhere inside Prandtl-Meyer wave $r_{3}$. The Eq. (40) is the special case of generalized Chester-Whitham theorem proven in Ref. [8].

Analyzing Eq. (39) for $\hat{N}_{1}=0$, we transformed it to the same Eq. (38) which was achieved earlier concerning wave $r_{3}$ change of type. The coincidence of the solutions subsequent to "differential" model (for the points situated immediately after the shock) and "integral" model (for the reflected wave) reveals that the flow type seems not to change along the second-family reflected characteristics, even in zone 5 .

Flow type change in the reflected wave can be illustrated by the influence of the expansion wave on the shock with initial "sonic" strength $J_{2}=J_{*}=8.282$ at $M_{1}=3$ (the integral curve $K K_{1}$ in Fig. 3). The sector $K K_{2}$ of this curve corresponds to the flow expansion in the reflected wave (from $M_{2}=1$ to $\left.M_{3}^{(\varphi)}=1.0208\right)$. The flow compression in the reflected wave begins at $M=2.860$ and $J=6.543$ (point $K_{2}$ ). Shock inflection point $K_{3}$ ( $M=2.572, J=4.258$ ) is situated in this sector. The Mach number in the reflected wave diminishes to $M_{3}^{(\varphi)}=1.0007$ at the end of the compression sector $K_{2} K_{4} \quad\left(M=2.089\right.$ and $J=2.016$ at point $\left.K_{4}\right)$. But the flow at the reflected compression wave does not decelerate to the critical speed because the new expansion sector begins after the point $K_{4}$ till the very shock degeneration into the weak discontinuity ( $J \rightarrow 1$ at $M=1.630$; point $K_{1}$ ). The Mach number in the reflected wave strives there to 1.0016 , and the integral strength $J_{3}$ of the reflected wave strives to 0.9981 . The change of the reflected wave type under the influence of the compression wave on the oblique shock occurs in an inverse order.

Comparing the data on the reflected wave obtained here with the analytical results in Ref. [13] where the 
sequence of the Prandtl-Meyer wave and the shock is considered without the interaction between them, we must conclude in the following way. The wave $r_{3}$ reflected as a result of the interaction of the wave $r_{1}$ (flow deflection angle $\beta_{1}$ ) with the shock $s_{2}$ (flow deflection angle $\beta_{2}$ ) always strives to reduce gradually the pressure and the total deflection angle downstream it and, consequently, these parameters behind the resulting wave $S_{4}\left(r_{4}\right)$, to the parameters after the sole shock (wave) in a flow with Mach number $M_{0}$ which deflects the stream on an angle equal to $\beta_{1}+\beta_{2}$. Basing on Ref. (38), we can consider this conclusion as proven analytically.

\subsection{The Degeneration of the Resulting Shocks and} Waves

The condition $J_{4}=1$ is introduced as supplementary to Eqs. (33) and (34) corresponds to the shock degeneration at the very first (upstream) characteristic of the Prandtl-Meyer fan $r_{1}$. The total deflection angle in the incoming waves $\beta_{1}+\beta_{2} \neq 0$ generally in this case. The overall angle of flow deflection far from the streamlined surface strives to $\beta_{1}+\beta_{2}$ asymptotically mainly due to the disturbances occurring at wave $r_{3}$ reflection from this surface.

Inasmuch as some sectors of the reflected wave $r_{3}$ realize the expansion flow, and some sectors the compression one, they also mutually compensate each other sometimes. Supposing both $J_{3}=1$ and $J_{4}=1$ in Eqs. (33) and (34), we define the special type of the interaction between the expansion wave and the oblique shock. These conditions determine the curves $h_{i}(i=1,2)$ in Figs. 3a and 3b. When the shocks which correspond to these curves degenerate to weak discontinuities, they leave mutually annihilating disturbances in the reflected wave contemporarily.

The curves $h_{i}$ begin at the above-mentioned points $F_{i}$. We can indicate the coordinates of the marginal left point $a$ of the curve $h_{2} \quad\left(M_{a}=2.230\right.$, $J_{a}=2.081$ ), the point $b$ of its intersection with the line $\varphi_{1}\left(M_{b}=2.506, J_{b}=4.611\right)$, the points $d$ ( $\left.M_{d}=1.464, \quad J_{d}=J_{*}\left(M_{d}\right)=1.804\right)$ and $e$ ( $M_{e}=3.013, J_{e}=J_{*}\left(M_{e}\right)=8.358$ ) where the curves $h_{i}$ finish at "sonic" line 1 . The integral curves of Eq. (32) outgoing the sector be of the curve $h_{2}$ have two intersection points with the curve $\varphi_{2}$. This means that the reflected wave $r_{3}$ which consists of two expansion sectors and a compression one can be divided mentally into two waves of unit strength.

\subsection{Mach Numbers Downstream the Shocks, Subsonic Flow Pockets}

Two contradictory factors influence on the variation of the Mach number $M^{\prime}$ immediately downstream the interacting shock. On the one hand, this curvilinear shock weakens inside the expansion fan, its strength $J$ diminishes, and that leads to a Mach number increase behind the shock. But, on the other hand, the Mach number upstream the shock decreases, and it leads to a decrease in the Mach number downstream. Computations show that Mach number just downstream the shock increases as a rule at large and moderate Mach numbers upstream and diminishes at small Mach numbers.

The equation $d M^{\prime} / d M=0$ analyzed together with Eqs. (21) and (32) which bind the shock strength and Mach numbers on its sides determines curve 6 (i.e., the line $k n$ in Fig. 3b) of the local Mach numbers maxima just behind the interacting shock. Here $M_{k}=1.127$, $J_{k}=1$. The coordinates of the point $n \quad\left(M_{n}=1.257\right.$, $J_{n}=1.376$ ) are the only real roots of the equations

$$
\begin{gathered}
(1-\varepsilon) M_{n}^{6}-(4-5 \varepsilon) M_{n}^{4}+(7-8 \varepsilon) M_{n}^{2}-(5-4 \varepsilon)=0, \\
J_{n}^{3}-(1+\varepsilon) J_{n}-1=0 .
\end{gathered}
$$

A local decrease in the downstream Mach number that corresponds to the region $O k n$ can lead to the flow transition downstream the shock through the critical speed ( $\left.K^{\prime}=1\right)$. It can become earlier than the shock wave degenerates into a weak discontinuity. So the integral curves of Eq. (32) situated in the area between "sonic" line 1 and dotted integral line 7, or $O g\left(M_{g}=1.391, J_{g}=1.640\right)$ both start and finish at 
the "sonic" line (to the right of point $g$ and to the left of it, correspondingly). Considering only the supersonic flow downstream the shock, we can conclude that the integral curve starting from point $n$ finishes at the same point at once.

When the shock $j_{2}$ interacts with the preceding expansion wave $r_{1}$, the shock strength diminishes, and full pressure losses at the interacting shock become smaller. The Mach number immediately behind the interacting shock is always larger than the Mach number at the corresponding characteristic of the reflected wave. So, when the flow immediately after the shock sometimes decelerates to a critical and even subsonic velocity, it means that the supersonic flow in the reflected mainly compression wave $r_{3}$ have decelerated to the critical velocity sooner.

According to the model Eqs. (33) and (34), the critical flow deceleration in the reflected compression wave is possible for incoming shocks which correspond to the dashed regions " $A$ " and " $B$ " adjacent to the curves $8 a$ (Fig. 3b) and $8 b$ (Fig. 3a) if the incoming expansion wave $r_{1}$ is strong enough. Points $d$ and $e$ at the "sonic" line whose coordinates are calculated in Subsection $3.4 \quad\left(M_{d}=1.464\right.$, $M_{e}=3.013$ ) are the endpoints of "A" and "B" regions, correspondingly. The region of possible flow deceleration immediately downstream the shock bounded by the integral curve $O g$ and "sonic" line 1 is situated inside the region " $\mathrm{A}$ " of flow deceleration in the reflected wave.

Possible solutions for the strong incoming shocks (at $J_{*}\left(M_{1}\right)<J_{2}<J_{m}(M)$, so $M_{2}<1$, Fig. 1d) ignore the influence of the other disturbances from the subsonic flow downstream the shock to this one. So it seems that the solutions for the strong shocks cannot be mentioned here due to their low practical importance.

\subsection{The Influence of Gas Specific Heats Ratio on the Problem Solution}

To characterize the influence of specific heats ratio on the solutions derived here, it seems enough to study the variation of the special curves and the points plotted above.

Starting points $F_{i}$ of the curves $\varphi_{i}$ merge at $\gamma \rightarrow 1 \quad\left(M_{F_{1}}=M_{F_{2}}=\sqrt{2}\right.$ then). A distance between these curves increases with the growth of $\gamma$. So the region II which corresponds to the expansion wave reflection at shock interaction with incoming expansion wave widens then.

The first of the start points $F_{i}$ moves to smaller Mach numbers region (so $M_{F_{1}}=1.245$ at $\gamma=1.4$, $M_{F_{1}}=1.189$ at $\gamma=3, M_{F_{1}} \rightarrow 2 / \sqrt{3}$ at $\left.\gamma \rightarrow \infty\right)$. The same is correct for the whole $\varphi_{1}\left(F_{1} s\right)$ line (so $M_{s} \rightarrow 1.318 \quad$ and $\quad J_{s} \rightarrow 1.434 \quad$ at $\quad \gamma \rightarrow 1$; $M_{s}=1.305$ and $J_{s}=1.466 \quad$ at $\quad \gamma=1.4$; $M_{s}=1.288$ and $J_{s}=1.529$ at $\gamma=3 ; M_{s} \rightarrow 1.272$ and $J_{s} \rightarrow 1.618$ at $\left.\gamma \rightarrow \infty\right)$ ). So region I where the reflected wave $r_{3}$ is of another type than incoming wave $r_{1}$ becomes narrower.

The curve $k n$ of the maximum downstream Mach numbers also exists at all $1<\gamma<\infty$ moving a little to the larger Mach numbers (so $M_{n} \rightarrow 1.253$ and $J_{n} \rightarrow 1.325 \quad$ at $\quad \gamma \rightarrow 1 \quad ; \quad M_{n}=1.257 \quad$ and $J_{n}=1.376$ at $\gamma=1.4 ; M_{n}=1.263$ and $J_{n}=1.567$ at $\gamma=3 ; M_{n} \rightarrow 1.272$ and $J_{n} \rightarrow 1.618$ at $\left.\gamma \rightarrow \infty\right)$. Points $n$ and $s$ coincide at $\gamma \rightarrow \infty$.

Another reflected wave type change curve $\varphi_{2}$ moves sufficiently to the right. So the coordinates of its extreme left point $u$ are the following: $M_{u}=1.790$ and $J_{u}=1.436$ at $\gamma=1.2 ; \quad M_{u}=2.089$ and $J_{u}=1.989$ at $\gamma=1.4 ; M_{u}=2.539$ and $J_{u}=2.980$ at $\gamma=1.66$. The Mach number $M_{F_{2}}$ also increases ( $M_{F_{2}}=1.876$ at $\gamma=1.2, M_{F_{2}}=2.540$ at $\gamma=1.4$, $M_{F_{2}}=16.325$ at $\left.\gamma=1.66\right)$ and strives to infinity at $\gamma=5 / 3$. The lower branch of the curve $\varphi_{2}$ has a horizontal asymptote $(J=1)$ in the last case. The further increase of the specific heats ratio leads to a corresponding shift of the curve $\varphi_{2}$ to the right (so $M_{u}=6.027$ and $J_{u}=16.098$ at $\gamma=3 ; M_{u} \rightarrow \infty$ and $J_{u} \rightarrow \infty$ at $\gamma \rightarrow \infty$ ). The curve $h_{2}$ and the region $3 b$ corresponding to shock degeneration and 
critical flow deceleration in the reflected waves also shift to the right. The rules determining the direction of shock curvature remain the same for other $\gamma$ 's. Mach numbers and shock strengths corresponding to the shock inflection increase a little: for example, $M_{r} \rightarrow 1.659$ and $J_{r} \rightarrow 2.206 \quad$ at $\quad \gamma \rightarrow 1$; $M_{r}=1.676 \quad$ and $J_{r} \rightarrow 2.361 \quad$ at $\gamma=1.4$; $M_{r} \rightarrow \sqrt{3}$ and $J_{r} \rightarrow 3$ at $\gamma \rightarrow \infty$. The inflection curve 2 always has the horizontal asymptote $J=J_{A}$ (e.g., $J_{A} \rightarrow 13.061$ at $\gamma \rightarrow \infty$ ).

So the increase in specific heats ratio widens the expansion wave reflection region (as $r_{1}$ is the expansion wave) and shifts the majority of the special curves and points plotted here to the region of larger Mach numbers and shock strengths.

The dependence of some specific Mach numbers upon the specific heats ratio in the practically important range $(1<\gamma<2)$ is shown in Fig. 5. One can see, for example, that some specific small Mach numbers ( $M_{k}$, $M_{n}, M_{s}, M_{g}, M_{d}, M_{r}$ ) are weakly dependent on $\gamma$ in the range shown there.

\section{Conclusions}

Owing to the expressions derived for the differential parameters of Prandtl-Meyer flowfield and the

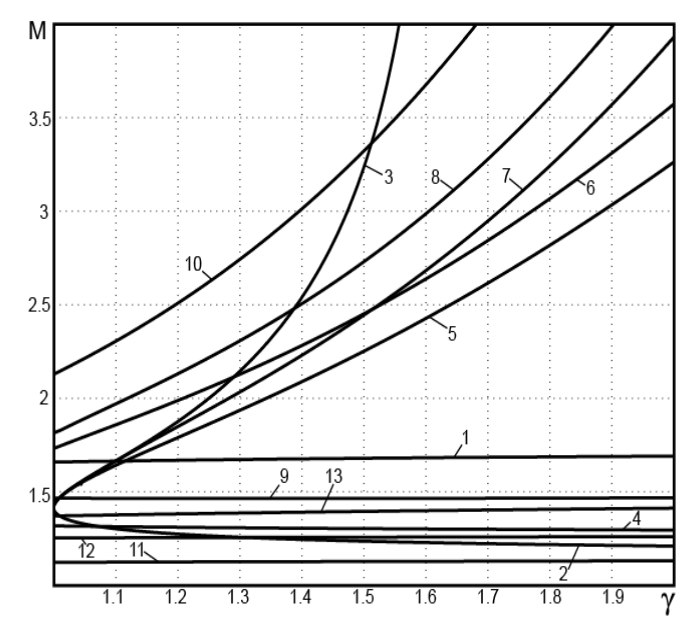

Fig. 5 Variation of the specific Mach numbers in the practically important range of $\gamma$ 's. According to the citation order: $M_{r}$ (curve 1), $M_{F_{1}}$ and $M_{F_{2}}$ (curves 2 and 3, correspondingly), $M_{s}$ (curve 4), $M_{u}$ (5), $M_{v}$ (6), $M_{a}$ (7), $M_{b}$ (8), $M_{d}$ (9), $M_{e}$ (10), $M_{k}$ (11), $M_{n}$ (12) and $M_{g}$ (13). differential dynamic compatibility conditions on a curved shock in a generally non-uniform flow, two reliable analytical solutions for overtaking Prandtl-Meyer wave-oblique shock interaction were obtained.

When used together, these solutions allows us to estimate both the flow parameters and their spatial derivatives downstream the shock as well as the distinctive features of the interacting shock and reflected Prandtl-Meyer wave. So the geometrical curvature and the inflection points at the interacting shock were determined analytically, in particular.

A simple expression determining the rarefaction/compression flow type change immediately behind the shock as well as in the reflected wave was derived also. It was proven that the reflected Prandtl-Meyer wave can change its type, not once somewhere. The criteria of the full mutual compensation of the different sectors of the reflected wave and the criteria of the shock degeneration were also derived and studied.

Non-monotonic variation of the Mach numbers just downstream the curvilinear interacting shock was discovered, and the possibility of subsonic flow pockets was revealed. The influence of the ratio of gas specific heats on the deduced solutions was found more quantitative than qualitatively remarkable.

The most evident applications of the presented results are: the optimal design of supersonic inlets; the design of optimally shaped aerodynamic bodies for supersonic flight; the analysis of supersonic jet technology apparatus because of the sequential interchange of compression and expansion flow regions divided by the shocks in the "barrels" of supersonic jet flows.

\section{References}

[1] R. Courant, K.O. Friedrichs, Supersonic Flow and Shock Waves, Wiley Interscience, New York, 1948.

[2] A.L. Adrianov, A.L. Starykh, V.N. Uskov, Interaction of Stationary Gas-Dynamic Discontinuities, Novosibirsk, Nauka, 1995. (in Russian)

[3] V.N. Uskov, M.V. Chernyshov, Conjugation of Prandtl-Meyer wave with quasi-one-dimensional flow 
region, Mathematical Modeling 15 (6) (2003) 111-119. (in Russian)

[4] G. Emanuel, H. Hekiri, Vorticity jump across a shock in nonuniform flow, Shock Waves 21 (1) (2011) 71-72.

[5] G. Emanuel, H. Hekiri, Vorticity and its rate of change just downstream of a curved shock, Shock Waves 17 (1-2) (2007) 85-94.

[6] A.V. Omelchenko, On the correlation of derivatives at a strong discontinuity, Computational Mathematics and Mathematical Physics 42 (8) (2002) 1246-1257. (in Russian)

[7] M.V. Chernyshov, The Interactions of the Elements of Shock-Wave Systems Between Themselves and with Different Surfaces, Ph.D. Thesis, Saint Petersburg, Baltic State Technical University, 2002. (in Russian)

[8] A.V. Omelchenko, The generalized Chester-Whitham invariant, Technical Physics Letters 27 (11) (2001) 891-893.
[9] A.L. Adrianov, A Model of the Flow Downstream the Curvilinear Stationary Shock, Krasnoyarsk, Computational Center of RAS Siberian Branch, 1997. (in Russian)

[10] H. Li, G. Ben-Dor, Oblique Shock-Expansion Fan Interaction-Analytical Solution, AIAA Journal 43 (2) (1996) 418-421.

[11] V.R. Meshkov, A.V. Omelchenko, V.N. Uskov, The interaction of the stationary shock with the expansion wave of opposite direction, Vestnik Saint Petersburg University: Mathematics 2 (9) (2002) 99-106. (in Russian)

[12] A.V. Omelchenko, V.N. Uskov, An optimal shock-expansion system in a steady gas flow, Journal of Applied Mechanics and Technical Physics 38 (3) (1997) 204-210.

[13] A.V. Omelchenko, V.N. Uskov, Optimum overtaking compression shocks with restriction imposed on the total flow-deflection angle, Journal of Applied Mechanics and Technical Physics 40 (4) (1999) 638-646. 